Relations industrielles

Industrial Relations

\title{
La Revue des relations industrielles
}

\section{Gérard Tremblay}

Volume 6, numéro 1, décembre 1950

URI : https://id.erudit.org/iderudit/1023244ar

DOI : https://doi.org/10.7202/1023244ar

Aller au sommaire du numéro

Éditeur(s)

Département des relations industrielles de l'Université Laval

ISSN

0034-379X (imprimé)

1703-8138 (numérique)

Découvrir la revue

Citer cet article

Tremblay, G. (1950). La Revue des relations industrielles. Relations industrielles /Industrial Relations, 6(1), 3-3. https://doi.org/10.7202/1023244ar

Tous droits réservés (C Département des relations industrielles de l’Université Laval, 1950
Ce document est protégé par la loi sur le droit d'auteur. L'utilisation des services d'Érudit (y compris la reproduction) est assujettie à sa politique d'utilisation que vous pouvez consulter en ligne.

https://apropos.erudit.org/fr/usagers/politique-dutilisation/ 


\section{La Revue des relations industrielles}

Le Département des relations industrielles de la Faculté des sciences sociales de Laval est fier de présenter d̀ ses lecteurs, sous une forme nouvelle et rajeunie, la Revue des relations industrielles qui remplace le Bulletin du même nom.

Pourquoi cette transformation dans la présentation de la Revue? D'abord, pour lui donner un peu plus de volume et permettre ainsi la publication d'études plus élaborées sur les sujets qui intéressent nos lecteurs. Les deux sections du Bulletin offraient vingt-quatre pages de texte; la Revue en comporte soixante. Il devient alors plus facile pour nos collaborateurs de mieux présenter un sujet, de le développer scientifiquement et d'exposer de façon plus critique leurs conclusions.

La Revue devient trimestrielle de mensuel qu'était le Bulletin. La direction pourra porter plus d'attention à une rédaction trimestrielle. Il ne faut pas oublier que la publication mensuelle du Bulletin imposait d̀ nos collaborateurs, déjà très pris par ailleırs, un fardeau devenant de plus en plus lourd si l'on songe que tous participent de façon bénévole au travail de rédaction. La publication trimestrielle, même si elle est de volume équivalent pour une année, permet une meilleure répartition du travail.

Le format paraîtra plus commode d̀ ceux de nos lecteurs qui nous font l'honneur de conserver les séries en bibliothèque, quatre éditions de soixante pages, si l'on tient compte du texte anglais, formant un joli volume annuel de deux cent quarante pages.

La Revue demeure bilingue. Elle est d'inspiration catholique et française à l'image de l'Université Laval dont elle est la collaboratrice humble et dévouée. Nous estimons, cependant, que son rayonnement est facilité par la version anglaise que nous essaierons daméliorer. Déjà près de la moitié de nos lecteurs sont de langue anglaise. Au surplus, les échanges avec les universités canadiennes et américaines permettent à la Revue de pénétrer dans des milieux qui lui resteraient étrangers si les textes étaient exclusivement français.

Nous croyons bonne l'occasion de remercier nos collaborateurs de ces cinq années. Ils ont abordé dans les cinquante numéros, formant six cents pages de texte français et anglais, la majeure partie des problemes de sociologie, d'économique, de droit et de relations de travail qui retiennent l'attention chez nous. Nous ne prétendons pas que le Bulletin ait été la réalisation des rêves de tous, mais on admettra qu'il a été un instrument utile et constant d'exposition loyale de la doctrine sociale catholique sans pour cela ignorer la nécessité des études positives sur notre milieu.

Merci d nos lecteurs que nous souhaitons toujours plus attentifs et nombreux. Inutile de souligner que nous recevrons d'eux, avec le meilleur esprit, toutes suggestions qu'ils croient opportunes d l'amélioration de notre publication.

Enfin, notre reconnaissance est acquise aux personnes qui ont eu l'obligeance d'aider matériellement notre oeuvre en souscrivant une publicité dont nous souhaitons qu'elle leur rende le centuple.

Notre mot de la fin: que cette humble revue continue de remplir vaillamment le rôle qui lui a été assigné d'affirmer et de propager le témoignage chrétien dans la recherche des solutions aux problemes si complexes de la vie économique et sociale de la patrie canadienne. 